\title{
Tratamiento ordinal de las escalas cualitativas utilizadas por el Centro de Investigaciones Sociológicas
}

\author{
GONZÁLEZ DEL POZO, RAQUEL \\ Departamento de Economía Aplicada \\ Universidad de Valladolid \\ Correo electrónico: raquel.gonzalez.pozo@uva.es \\ GARCÍA LAPRESTA, JOSÉ LUIS \\ Departamento de Economía Aplicada \\ Universidad de Valladolid \\ Correo electrónico: lapresta@eco.uva.es
}

\begin{abstract}
RESUMEN
Las escalas cualitativas formadas por términos lingüísticos son utilizadas por diversas disciplinas para determinar preferencias y diversos aspectos de la vida de los individuos. Aunque es habitual asignar números a las categorías de respuesta de estas escalas, no es adecuado utilizar codificaciones numéricas cuando los individuos perciben proximidades psicológicas distintas entre las categorías consecutivas de la escala, es decir, cuando las escalas cualitativas no son uniformes.

En este trabajo se propone un procedimiento ordinal para jerarquizar un conjunto de alternativas a partir de las valoraciones mostradas por un grupo de individuos mediante una escala cualitativa no necesariamente uniforme. Dicho procedimiento está basado en las proximidades ordinales entre las categorías de respuesta de las escalas. El procedimiento propuesto se ilustra con un ejemplo tomado del Barómetro del Centro de Investigaciones Sociológicas (CIS) de mayo de 2011.
\end{abstract}

Palabras clave: encuestas, cuestionarios, escalas cualitativas, imprecisión, CIS.

Clasificación JEL: C20; C83.

MSC2010: 62C12; 62C86. 


\title{
Ordinal treatment of qualitative scales used by the Center for Sociological Research
}

\begin{abstract}
Qualitative scales formed by linguistic terms are used by different disciplines to determine preferences and different aspects of individuals' lives. Although it is usual to assign numbers to the response categories of scales, it is not suitable when individuals perceive different proximities between the consecutive categories of the scale, that is, when scales are not uniform.

In this paper, an ordinal procedure is proposed to order a set of alternatives from the assessments given by a group of individuals through a qualitative scale not necessarily uniform. This procedure is based on ordinal proximities between the response categories of scales. The proposed procedure is illustrated with an example taken from the Barometer of the Center for Sociological Research of May 2011.
\end{abstract}

Keywords: surveys, questionnaires, qualitative scales, imprecision, CIS.

JEL classification: C20; C83.

MSC2010: 62C12; 62C86

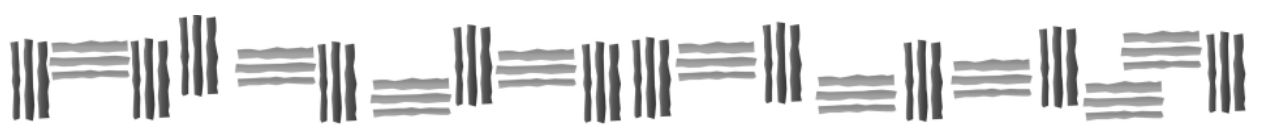




\section{Introducción.}

El creciente interés por conocer y evaluar las preferencias, opiniones y necesidades de la población hace que disciplinas como Sociología, Economía, Psicología, Marketing o Medicina elaboren rankings o listas que tienen por objeto la jerarquización de un conjunto de alternativas (hoteles, países, instituciones de educación superior, etc.).

Diversos autores (Beyth-Marom, 1982; Zimmer, 1983; Teigen \& Wibecke, 1999; Durán, Ocaña, Cañadas \& Pérez, 2000, entre otros) han señalado que los seres humanos se sienten más cómodos utilizando palabras en lugar de valores numéricos para manifestar sus opiniones y valoraciones, ya que las respuestas verbales suponen un menor tiempo de procesamiento y evaluación de las mismas, permitiéndoles, además, expresarse de una forma sencilla ante situaciones de vaguedad e imprecisión.

Debido a esta preferencia de los individuos por las palabras, es frecuente que los cuestionarios y encuestas incorporen escalas cualitativas para conocer opiniones, percepciones o juicios subjetivos de los encuestados. Es el caso, por ejemplo, de la Encuesta de Condiciones de Vida (ECV) o European Union Statistics on Income and Living Conditions (EU-SILC en terminología europea), que utiliza la siguiente escala cualitativa \{ "Una carga pesada”, "Una carga razonable", "Ninguna carga” $\}$ para preguntar a los hogares objeto de estudio qué tipo de carga les suponen los gastos o desembolsos relacionados con compras a plazo o devolución de préstamos. Esta misma encuesta también emplea una escala cualitativa de seis categorías de repuesta para preguntar a los hogares cómo llegan a fin de mes: ‘"Con mucha dificultad", "Con dificultad", "Con cierta dificultad", "Con cierta facilidad", "Con facilidad”, "Con mucha facilidad”\}.

Por otra parte, el Estudio Internacional de Tendencias en Matemáticas y Ciencias, conocido internacionalmente como The Trends in International Mathematics and Science Study (TIMSS), evalúa a través de varios cuestionarios dirigidos a alumnos, familias, profesorado y centros escolares el rendimiento en ambas competencias de los alumnos de cuarto curso de Educación Primaria y segundo curso de Educación Secundaria Obligatoria. Para esta evaluación el TIMSS incorpora en sus cuestionarios diversas escalas cualitativas. Así, en el cuestionario dirigido a los centros para valorar diversos problemas escolares como el absentismo, el vandalismo o las peleas escolares, se utiliza la escala \{ "Problema severo", "Problema moderado", "Problema menor”, "No es un problema”\}.

Asimismo, el Centro de Investigaciones Sociológicas (CIS) también introduce en sus cuestionarios escalas cualitativas cuyas respuestas oscilan entre tres y cinco categorías. Ejemplo de ello es la escala cualitativa de cuatro términos \{ "Nada", "Poco", "Bastante”, "Mucho"\}, usada frecuentemente por el CIS en sus Barómetros para conocer las opiniones y valoraciones de los encuestados sobre diversos temas sociales, políticos y culturales.

En los cuestionarios también es frecuente encontrar escalas tipo Likert (Likert, 1932) para conocer el grado de acuerdo o desacuerdo de los encuestados ante determinadas cuestiones, así como para recoger información acerca de sus actitudes y preferencias. Los encuestados realizan sus valoraciones a través de una escala cualitativa ordenada cuyas categorías de respuesta varían normalmente entre tres y siete términos, si bien algunos autores sugieren que el número óptimo de categorías a introducir en las escalas Likert debe estar comprendido entre cuatro y siete (Lozano, García-Cueto \& Muñiz, 2008; Nadler, Weston \& Voyles, 2015).

Habitualmente este tipo de escalas son simétricas, es decir, tienen el mismo número de categorías favorables y desfavorables, separadas por una categoría de respuesta central. La introducción de esta categoría intermedia es objeto de controversia en la elaboración de cuestionarios, al considerar que recoge gran parte de las respuestas de los encuestados que no quieren manifestar sus opiniones de forma explícita (Díaz de Rada, 2015; Nadler, Weston \& 
Voyles, 2015) y puede llegar a provocar la aparición de un sesgo de tendencia central, si la mayoría de las respuestas de los encuestados se concentran en ella (Mangione, 1995; Matas, 2018, entre otros).

Por regla general es frecuente asignar valores numéricos (normalmente equidistantes) a las categorías de respuesta de las escalas cualitativas. Sin embargo, evidencias empíricas han demostrado que, en ocasiones, pueden apreciarse proximidades psicológicas distintas entre las categorías de repuesta de las escalas (Kennedy, Riquier \& Sharp, 1996), es decir, las escalas cualitativas pueden percibirse como no uniformes. Así pues, la escala cualitativa del CIS mencionada anteriormente podrá ser considerada no uniforme si los encuestados perciben que la categoría "Bastante” está más próxima a "Mucho" que a "Poco”, o si la categoría "Poco” está más próxima a "Nada” que a "Bastante".

Al diseñar encuestas es importante considerar la no uniformidad de las escalas cualitativas para evitar errores de medida y de no respuesta parcial y/o total durante el proceso de recogida de datos (Díaz de Rada, 2004). De igual forma, conviene destacar la importancia que juega el contexto en el que se aplica la escala cualitativa a la hora de determinar las proximidades psicológicas entre los términos de la misma, ya que éste puede influir significativamente en la forma que tienen los encuestados de concebir dichas proximidades.

Cuando las escalas cualitativas son consideradas como no uniformes, la codificación numérica de las categorías de respuesta puede no reflejar adecuadamente las diferencias que aprecian los encuestados entre cada una de ellas (Silva, 1997; Fleiss, Levin \& Paik, 2003). Asimismo, estas codificaciones pueden dificultar la representatividad e interpretación de los resultados obtenidos (Stevens, 1951; Pardo, 2002), ya que estos pueden variar en función de la codificación utilizada (Roberts, 1979; Merbitz, Morris \& Grip, 1989; Franceschini, Galetto \& Varetto, 2004, entre otros).

La asignación de números a las categorías de respuesta no es el único aspecto a considerar en el contexto de las escalas cualitativas no uniformes, siendo también importante cómo se trata y presenta la información procedente de dichas escalas.

Diversos organismos nacionales e internacionales suelen publicar los resultados de sus investigaciones agrupándolos en determinadas categorías de respuesta (por ejemplo "Mucho" y "Bastante" o "Very good" y "Somewhat good"). La agrupación de varias categorías de respuesta puede encontrarse en los anuarios publicados por el CIS, y en algunos informes del Centre d'Estudis d'Opinió (CEO) de Cataluña, del Centro de Análisis y Documentación Política y Electoral de Andalucía (CADPEA), de la Fundación Española para la Ciencia y la Tecnología (FECYT) y del Pew Research Center de Estados Unidos, entre otros. No obstante, esta práctica no está considerando la no uniformidad de las escalas cualitativas y además supone una pérdida importante de la información cualitativa de las mismas.

Así, por ejemplo, cuando el CIS agrupa en sus anuarios las categorías "Bastante” y "Mucho" y descarta las respuestas de las categorías "Nada” y "Poco" está asignando de forma implícita un mismo valor numérico a las categorías de respuesta agrupadas y una puntuación nula a las categorías omitidas. Por ejemplo, en cada alternativa a evaluar se estarían asignando 0 puntos a cada respuesta "Nada" o "Poco" y 1 punto a cada respuesta "Bastante” o "Mucho".

Para ilustrar este problema consideraremos, a modo de ejemplo, dos alternativas A y B, que han sido valoradas utilizando la escala cualitativa del CIS. En la Tabla 1 se muestran las valoraciones recibidas, expresadas en porcentajes. 
Tabla 1. Porcentajes de respuestas de las alternativas A y B.

\begin{tabular}{lcccc}
\hline & Mucho & Bastante & Poco & Nada \\
Alternativa A & 0 & 97 & 0 & 3 \\
Alternativa B & 96 & 0 & 4 & 0 \\
\hline
\end{tabular}

Fuente: Elaboración propia.

De acuerdo con la práctica de agrupación de varias categorías de respuesta, A vence a B, pues A ha obtenido un total de $97 \%$ de las respuestas "Mucho" y "Bastante”, mientras que B solo ha obtenido un $96 \%$. Sin embargo, una mínima compensación otorgaría ventaja a B sobre A, ya que la alternativa A no ha conseguido ninguna respuesta en la categoría "Mucho", mientras que la alternativa B ha conseguido un $96 \%$. Además, las respuestas obtenidas por la alternativa A en las categorías inferiores de la escala son todas "Nada", mientras que las de la alternativa B son mejores, al conseguir un $4 \%$ de respuestas en la categoría "Poco".

Para evitar resultados como los del ejemplo anterior, consecuencia del tratamiento inapropiado de la información procedente de escalas cualitativas, en este trabajo se plantea jerarquizar un conjunto de alternativas valoradas cualitativamente a través del procedimiento puramente ordinal propuesto recientemente por García-Lapresta y Pérez-Román (2018). Este procedimiento puede aplicarse en el contexto de las escalas cualitativas no uniformes, ya que se basa en cómo aprecian los individuos las proximidades entre las categorías de respuesta de las escalas cualitativas, sin necesidad de asignar valores numéricos u otros objetos matemáticos a los términos lingüísticos de las mismas.

El objetivo del presente trabajo es analizar críticamente la metodología de agrupación de varias categorías de respuesta que realiza el CIS (así como otros organismos) y considerar como método alternativo el procedimiento ordinal mencionado. Para ello, hemos realizado un análisis comparativo de los órdenes (rankings) resultantes de aplicar el procedimiento ordinal antes mencionado y la práctica de agrupación de varias categorías de respuesta.

El trabajo se encuentra organizado como sigue: en primer lugar, se introducen brevemente el concepto de medida de proximidad ordinal y el procedimiento ordinal (García-Lapresta y Pérez-Román, 2018). En segundo lugar, se aplica el procedimiento ordinal para jerarquizar un conjunto de alternativas procedentes del Barómetro del CIS. Los resultados obtenidos se comparan con los obtenidos mediante la práctica de agrupación de varias categorías de respuesta. Finalmente, el trabajo termina con algunas consideraciones y conclusiones sobre el uso de escalas cualitativas.

\section{Metodología.}

Con objeto de considerar toda la información que nos proporcionan las escalas cualitativas y evitar el uso de codificaciones numéricas arbitrarias que no reflejen adecuadamente las proximidades entre las categorías de respuesta de las escalas, en este trabajo proponemos la aplicación del procedimiento ordinal introducido por García-Lapresta y Pérez-Román (2018). Dicho procedimiento está basado en la noción de medida de proximidad ordinal.

\section{Concepto de medida de proximidad ordinal}

El concepto de medida de proximidad ordinal introducido por García-Lapresta y Pérez-Román (2015) recoge la información sobre cómo aprecian los individuos las proximidades entre las categorías de respuesta de una escala cualitativa, cuestión de máxima relevancia cuando las escalas cualitativas son consideradas como no uniformes. 
Para poder definir una medida de proximidad ordinal es necesario partir de una escala cualitativa ordenada $\mathcal{L}=\left\{l_{1}, \ldots, l_{g}\right\}$, donde $l_{1} \prec \cdots \prec l_{g}$ y $g \geq 3$. Sirva como ejemplo la escala que aparece en la Tabla 2, utilizada en la ECV.

Tabla 2. Categorías de respuesta de la escala utilizada por la ECV.

\begin{tabular}{cl}
\hline$l_{3}$ & Ninguna carga \\
$l_{2}$ & Una carga razonable \\
$l_{1}$ & Una carga pesada \\
\hline
\end{tabular}

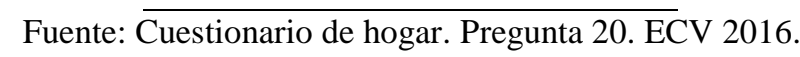

Asimismo, para generar una medida de proximidad ordinal es necesario un conjunto ordenado de grados de proximidad $\Delta=\left\{\delta_{1}, \ldots, \delta_{h}\right\}$, donde $\delta_{1}>\cdots>\delta_{h}$ ( $\delta_{1}$ es la máxima proximidad y $\delta_{h}$ la mínima, ocupando el resto de grados posiciones intermedias, ordenadas de mayor a menor proximidad). Es importante destacar que los elementos de $\Delta$ no son números, sino grados de proximidad, es decir, aportan información relativa sobre cuán próximas están unas categorías de otras, pero no representan números ni distancias entre las categorías de respuesta de las escalas cualitativas.

Una medida de proximidad ordinal sobre $\mathcal{L}$ con valores en $\Delta$ asigna a cada par de términos de la escala $l_{r}$ y $l_{s}$ un grado de proximidad $\pi_{r s}$ en $\Delta$, de forma que se cumplan las siguientes condiciones:

1. Todos los grados de proximidad de $\Delta$ son utilizados al menos una vez. Es decir, para cualquier grado de proximidad $\delta_{k}$, existen al menos dos términos de la escala, $l_{r}$ y $l_{s}$, tales que $\pi_{r s}=\delta_{k}$.

2. El grado de proximidad entre dos términos de la escala, $l_{r}$ y $l_{s}$, no depende del orden en el que se comparen, es decir, $\pi_{r s}=\pi_{s r}$.

3. La máxima proximidad entre términos de la escala, $\delta_{1}$, solo se alcanza cuando se compara un término consigo mismo. Es decir, $\pi_{r s}=\delta_{1}$ si y solo si $r=s$.

4. Si $r<s<t$, entonces $l_{r}$ está más cerca de $l_{s}$ que de $l_{t}$, y $l_{s}$ está más cerca de $l_{t}$ que $l_{r}$ de $l_{t}$, es decir $\pi_{r s}>\pi_{r t}$ y $\pi_{s t}>\pi_{r t}$ (véase la Figura 1).

Figura 1. Condición 4.

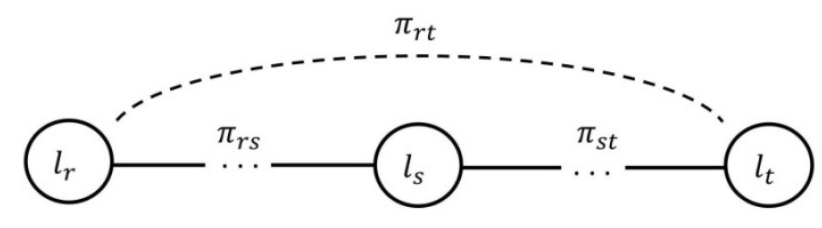

Fuente: Elaboración propia.

A modo de ejemplo, se ilustran gráficamente en la Figura 2 las tres medidas de proximidad ordinal que pueden generarse en escalas cualitativas formadas por tres categorías de respuesta. 
Figura 2. Medidas de proximidad ordinal para escalas cualitativas ordenadas formadas por tres categorías de respuesta.

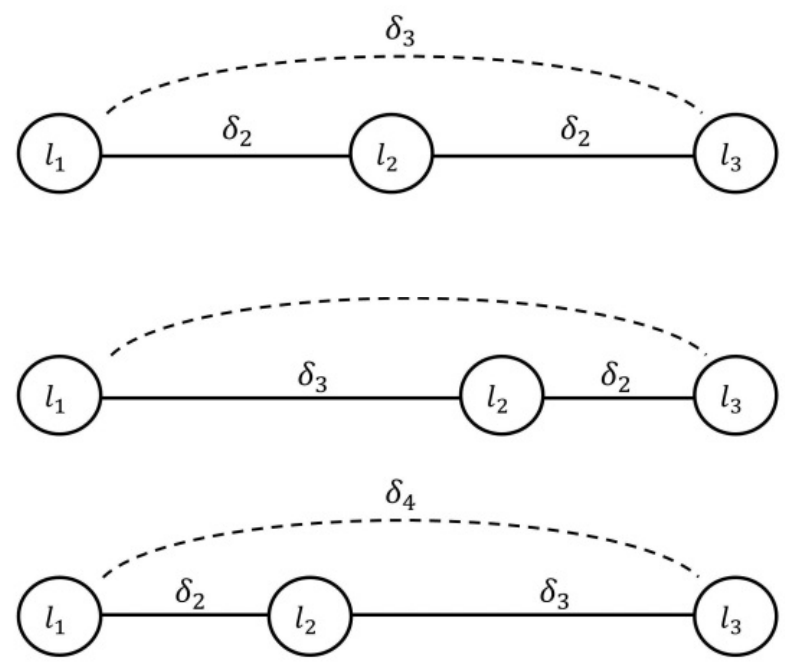

Fuente: Elaboración propia.

\section{Procedimiento ordinal}

Se considerará un conjunto de individuos $A=\{1, \ldots, m\}$, con $m \geq 2$, que evalúan un conjunto de alternativas $X=\left\{x_{1}, \ldots, x_{n}\right\}, \operatorname{con} n \geq 2 \quad$ a través de una escala cualitativa $\mathcal{L}=\left\{l_{1}, \ldots, l_{g}\right\}$, donde $l_{1} \prec \cdots \prec l_{g}$ y $g \geq 3$.

Las valoraciones dadas por los individuos a cada una de las alternativas son recogidas en una matriz de términos lingüísticos

$$
\left(\begin{array}{ccccc}
v_{1}^{1} & \cdots & v_{i}^{1} & \ldots & v_{n}^{1} \\
\cdots & \cdots & \cdots & \ldots & \cdots \\
v_{1}^{a} & \cdots & v_{i}^{a} & \cdots & v_{n}^{a} \\
\cdots & \ldots & \cdots & \cdots & \cdots \\
v_{1}^{m} & \ldots & v_{i}^{m} & \cdots & v_{n}^{m}
\end{array}\right)
$$

donde $v_{i}^{a} \in \mathcal{L}$ es la valoración cualitativa dada por el individuo $a \in A$ a la alternativa $x_{i} \in X$.

Para la aplicación del procedimiento ordinal deben seguirse los siguientes pasos:

1. Establecer una medida de proximidad ordinal adecuada.

Para fijar una medida de proximidad ordinal sobre una escala cualitativa debe conocerse cómo aprecian los individuos las proximidades entre los términos de la escala. Es importante comunicar siempre a los individuos el contexto de la pregunta en la que se está utilizando la escala cualitativa (político, económico, sanitario, etc.), ya que el contexto social e incluso la formulación de las preguntas juegan un papel fundamental a la hora de determinar estas proximidades. Por ejemplo, la percepción entre "Nada" y "Poco" puede variar dependiendo de si se pregunta con qué frecuencia se realizan visitas culturales o con qué frecuencia se consumen sustancias estupefacientes.

Una vez indicado el contexto, la medida de proximidad ordinal puede venir determinada por un experto en el ámbito o disciplina en la que se utilice la escala cualitativa objeto de estudio, 
o bien fijarse agregando las opiniones de varios individuos sobre las proximidades entre los términos de la escala cualitativa (García-Lapresta, González del Pozo \& Pérez-Román, 2018).

2. Para cada alternativa $x_{i} \in X$ se calculan los grados de proximidad entre las valoraciones cualitativas dadas por los individuos y la máxima valoración posible de la escala, $l_{g}$ :

$$
\pi\left(v_{i}^{1}, l_{g}\right), \ldots, \pi\left(v_{i}^{m}, l_{g}\right) \in \Delta .
$$

3. Ordenar para cada alternativa $x_{i} \in X$ los grados de proximidad de forma decreciente (de mayor a menor proximidad) y seleccionar para cada alternativa su par de medianas, $M_{i}$.

Los grados de proximidad obtenidos en el paso anterior pueden sintetizarse a través de una medida de tendencia central. Puesto que los grados de proximidad no son números y, por tanto, no es posible realizar con ellos operaciones algebraicas, quedan excluidas como medidas de tendencia central las medias (aritmética, geométrica, armónica, ponderadas, etc.). Dada la naturaleza cualitativa de los grados de proximidad, se considerarán como medidas de tendencia central las medianas.

El sistema de votación Majority Judgment (Balinski \& Laraki, 2011) utiliza la mediana como elemento representativo de las valoraciones de los individuos. En este sistema los individuos valoran un conjunto de alternativas a través de una escala cualitativa ordenada. A continuación, se ordenan las valoraciones y se selecciona de cada alternativa la valoración cualitativa que ocupa la posición mediana (mediana baja en el caso de que el número de valoraciones sea par). No obstante, tomar únicamente el valor de la mediana baja supone una pérdida importante de información. Para evitar este problema, el procedimiento aplicado en este trabajo utiliza dos medianas. Así,

- Cuando el número de grados de proximidad es par, se consideran las dos medianas, $M_{i}=$ $\left(\delta_{r}, \delta_{s}\right)$, con $r, s \in\{1, \ldots, h\}$ tales que $r \leq s$.

Por ejemplo, a $\left(\delta_{1}, \delta_{1}, \delta_{2}, \delta_{3}\right)$ le correspondería $\left(\delta_{1}, \delta_{2}\right)$.

- Cuando el número de grados de proximidad es impar, se duplica la mediana. Por tanto, $M_{i}=\left(\delta_{r}, \delta_{r}\right)$, para algún $r \in\{1, \ldots, h\}$.

Por ejemplo, a $\left(\delta_{1}, \delta_{2}, \delta_{3}\right)$ le correspondería $\left(\delta_{2}, \delta_{2}\right)$.

Por consiguiente, $M_{i} \in \Delta_{2}$, donde $\Delta_{2}$ es el conjunto de medianas factibles:

$$
\Delta_{2}=\left\{\left(\delta_{r}, \delta_{s}\right) \in \Delta^{2} \mid r \leq s\right\}
$$

Es importante destacar que el procedimiento ordinal aplicado en este trabajo recoge la no uniformidad de las escalas cualitativas a través de los grados de proximidad, aspecto no considerado en el sistema de votación Majority Judgment.

4. Establecer un orden lineal apropiado sobre $\Delta_{2}$ para ordenar los pares de medianas de los grados de proximidad.

En este trabajo hemos considerado el orden lineal introducido por García-Lapresta y PérezRomán (2018):

$$
\begin{aligned}
\left(\delta_{r}, \delta_{s}\right) \succcurlyeq_{2}\left(\delta_{t}, \delta_{u}\right) \Leftrightarrow\left\{\begin{array}{l}
r+s<t+u \\
0 \\
r+s=t+u \quad \text { y } \quad s-r \leq u-t
\end{array}\right. \\
\text { para cualesquiera }\left(\delta_{r}, \delta_{s}\right),\left(\delta_{t}, \delta_{u}\right) \in \Delta_{2} .
\end{aligned}
$$


Este orden lineal está basado en la aversión a la incertidumbre de los individuos (Epstein, 1999). No obstante, éste no es el único orden lineal posible, pudiendo establecerse distintas formas de ordenar los pares de medianas si se estima oportuno.

5. Ordenar las alternativas teniendo en cuenta el orden débil sobre $X$ definido como $x_{i} \geqslant x_{j} \Leftrightarrow M_{i} \geqslant_{2} M_{j}$.

A veces es posible que algunas de las alternativas tengan el mismo par de medianas de grados de proximidad y estén, por tanto, empatadas. En estos casos puede aplicarse un procedimiento secuencial de desempate basado en Balinski y Laraki (2007), que consiste en eliminar el par de medianas de las alternativas que se encuentran en la situación de empate, calcular de nuevo el par de medianas entre los grados de proximidad restantes y volver a ordenar las alternativas según el orden establecido en el paso 4. Este procedimiento de desempate se repetiría hasta romper todos los empates y ordenar todas las alternativas.

En el Anexo se incluye un ejemplo que ilustra el procedimiento explicado en esta sección.

\section{Aplicación del procedimiento ordinal a las escalas cualitativas utilizadas en los barómetros del CIS.}

El CIS utiliza frecuentemente en sus cuestionarios la escala cualitativa que aparece en la Tabla 3 para determinar, entre otras cosas, los temas de mayor interés para los españoles, medir el grado de influencia de las nuevas tecnologías en la vida cotidiana o conocer el grado de confianza que inspira a los españoles determinados colectivos o instituciones.

Con objeto de ilustrar el procedimiento ordinal de la sección 2 y comparar sus resultados con los obtenidos mediante el método de agrupación de varias categorías de respuesta, en esta sección jerarquizaremos un conjunto de alternativas valoradas cualitativamente.

Para poder apreciar mejor los cambios que se producen al ordenar las diferentes alternativas a través de los dos métodos mencionados, hemos seleccionado una pregunta compuesta por un conjunto amplio de ítems. En concreto hemos utilizado datos procedentes de la pregunta 20 del Barómetro del CIS de mayo de 2011 (estudio 2.888), en la que se pide a los encuestados que manifiesten, a través de la escala cualitativa de la Tabla 3 , hasta qué punto confiarían en la ayuda que les puedan proporcionar las nueve alternativas contenidas en la Tabla 4 ante una situación de catástrofe o desastre.

Tabla 3. Categorías de respuesta de la escala utilizada por el CIS.

\begin{tabular}{cc}
\hline$l_{4}$ & Mucho \\
$l_{3}$ & Bastante \\
$l_{2}$ & Poco \\
$l_{1}$ & Nada \\
\hline \multicolumn{2}{c}{ Fuente: Barómetros del CIS. }
\end{tabular}

Aunque inicialmente el número de encuestados en el Barómetro de mayo de 2011 ascendía a 2.482 (1.218 hombres y 1.264 mujeres), en el presente trabajo la muestra utilizada se ha reducido a 2.219 individuos al eliminar las categorías de respuesta "No sabe” y "No contesta". Los porcentajes de respuesta de los 1.114 hombres y las 1.105 mujeres que componen la muestra utilizada aparecen recogidos en la Tabla 5. 
Tabla 4. Alternativas a valorar en la pregunta 20.

La Guardia Civil
Las ONGs
Los bomberos
Protección Civil
La policía
El ejército
Sus familiares
Sus vecinos
Sus amigos
Fuente: Barómetro de mayo de 2011. Estudio CIS 2.888.

Tabla 5. Porcentaje de respuestas a la pregunta 20 del Barómetro del CIS de mayo de 2011.

\begin{tabular}{|c|c|c|c|c|c|}
\hline \multicolumn{6}{|c|}{ Respuesta a la pregunta 20 (hombres y mujeres) } \\
\hline & Mucho & Bastante & Poco & Nada & $(\mathrm{N})$ \\
\hline La Guardia Civil & 32,04 & 49,89 & 15,01 & 3,06 & 2.219 \\
\hline Las ONGs & 22,89 & 51,33 & 20,32 & 5,45 & 2.219 \\
\hline Los bomberos & 48,85 & 46,24 & 4,15 & 0,77 & 2.219 \\
\hline Protección Civil & 38,04 & 51,69 & 8,88 & 1,40 & 2.219 \\
\hline La policía & 30,51 & 51,64 & 15,86 & 1,98 & 2.219 \\
\hline El ejército & 38,35 & 49,12 & 10,86 & 1,67 & 2.219 \\
\hline Sus familiares & 55,39 & 39,75 & 4,28 & 0,59 & 2.219 \\
\hline Sus vecinos & 29,43 & 49,98 & 17,62 & 2,97 & 2.219 \\
\hline Sus amigos & 40,78 & 50,25 & 8,11 & 0,86 & 2.219 \\
\hline \multicolumn{6}{|c|}{ Respuesta a la pregunta 20 (hombres) } \\
\hline & Mucho & Bastante & Poco & Nada & $(\mathrm{N})$ \\
\hline La Guardia Civil & 33,21 & 47,58 & 15,35 & 3,86 & 1.114 \\
\hline Las ONGs & 21,45 & 48,38 & 23,16 & 7,00 & 1.114 \\
\hline Los bomberos & 49,91 & 44,97 & 4,31 & 0,81 & 1.114 \\
\hline Protección Civil & 37,70 & 50,81 & 9,78 & 1,71 & 1.114 \\
\hline La policía & 30,43 & 50,18 & 17,15 & 2,24 & 1.114 \\
\hline El ejército & 39,23 & 47,40 & 11,40 & 1,97 & 1.114 \\
\hline Sus familiares & 55,66 & 39,14 & 4,76 & 0,45 & 1.114 \\
\hline Sus vecinos & 29,53 & 48,29 & 19,03 & 3,14 & 1.114 \\
\hline Sus amigos & 41,38 & 49,46 & 8,35 & 0,81 & 1.114 \\
\hline
\end{tabular}




\begin{tabular}{lccccc}
\multicolumn{7}{c}{ Respuesta a la pregunta 20 (mujeres) } & & \\
& Mucho & Bastante & Poco & Nada & $(\mathrm{N})$ \\
La Guardia Civil & 30,86 & 52,22 & 14,66 & 2,26 & 1.105 \\
Las ONGs & 24,34 & 54,30 & 17,47 & 3,89 & 1.105 \\
Los bomberos & 47,78 & 47,51 & 3,98 & 0,72 & 1.105 \\
Protección Civil & 38,37 & 52,58 & 7,96 & 1,09 & 1.105 \\
La policía & 30,59 & 53,12 & 14,57 & 1,72 & 1.105 \\
El ejército & 37,47 & 50,86 & 10,32 & 1,36 & 1.105 \\
Sus familiares & 55,11 & 40,36 & 3,80 & 0,72 & 1.105 \\
Sus vecinos & 29,32 & 51,67 & 16,20 & 2,81 & 1.105 \\
Sus amigos & 40,18 & 51,04 & 7,87 & 0,90 & 1.105 \\
\hline
\end{tabular}

Fuente: Elaboración propia a partir del estudio CIS 2.888.

Como ya se mencionó en la sección 2, para poder aplicar el procedimiento ordinal es necesario establecer una medida de proximidad ordinal adecuada. Para ello, se realizó una encuesta dirigida a un grupo de 20 estudiantes de cuarto curso del grado en Economía de la Universidad de Valladolid. En dicha encuesta se les preguntó a los estudiantes cómo apreciaban las proximidades entre los términos "Nada", "Poco", "Bastante" y "Mucho" en relación a la pregunta utilizada por el CIS en su Barómetro de mayo de 2011. Para evitar inconsistencias a la hora de determinar las correspondientes medidas de proximidad ordinal, la encuesta se diseñó siguiendo el algoritmo introducido por (García-Lapresta, González del Pozo \& Pérez-Román, 2018). Dicho algoritmo comienza comparando las proximidades $\pi_{12}$ y $\pi_{23}$, que en el caso de la escala considerada consiste en preguntar sobre las proximidades entre el término "Poco" y los términos "Nada” y "Bastante”. Según cómo sea la respuesta recibida ("Poco" está más cerca de "Nada" que de "Bastante", "Poco" está más cerca de "Bastante" que de "Nada", o las proximidades entre "Poco" y "Nada” y "Poco" y "Bastante" son equivalentes), el algoritmo mencionado genera una segunda pregunta, y así sucesivamente hasta obtener la medida de proximidad ordinal que refleje fielmente las percepciones de los encuestados sobre la escala.

De los resultados de la encuesta surgieron cinco medidas de proximidad distintas, es decir, cinco formas distintas de concebir las proximidades entre los términos de la escala cualitativa. Es importante destacar que un $94,10 \%$ de los estudiantes percibió la escala del CIS como no uniforme. La representación gráfica de las medidas de proximidad ordinal obtenidas y los porcentajes de encuestados que percibieron dichas medidas están recogidos en la Tabla 6.

Una vez que obtenidas las medidas de proximidad ordinal de los estudiantes, es necesario determinar una que represente de la mejor forma posible las opiniones de los individuos sobre las proximidades entre las categorías de respuesta de la escala. La elección del procedimiento de agregación de esta información es algo crucial, ya que pueden llegar a obtenerse resultados distintos e incluso inconsistencias, dependiendo del procedimiento de agregación utilizado.

Con objeto de establecer una medida de proximidad ordinal apropiada y evitar los problemas e inconsistencias considerados por la teoría de agregación de juicios (Judgment Aggregation) (véase, por ejemplo, Grossi \& Pigozzi, 2014), se ha utilizado un procedimiento basado en métricas ponderadas. Este procedimiento considera que la medida de proximidad ordinal que mejor representa las opiniones de los encuestados es aquella que minimice la suma de distancias (o la suma de los cuadrados de las distancias) entre ella y las medidas de proximidad ordinal de los individuos (García-Lapresta, González del Pozo \& Pérez-Román, 2018). 
Tabla 6. Resultados de la encuesta.

Representación gráfica de las medidas de proximidad ordinal

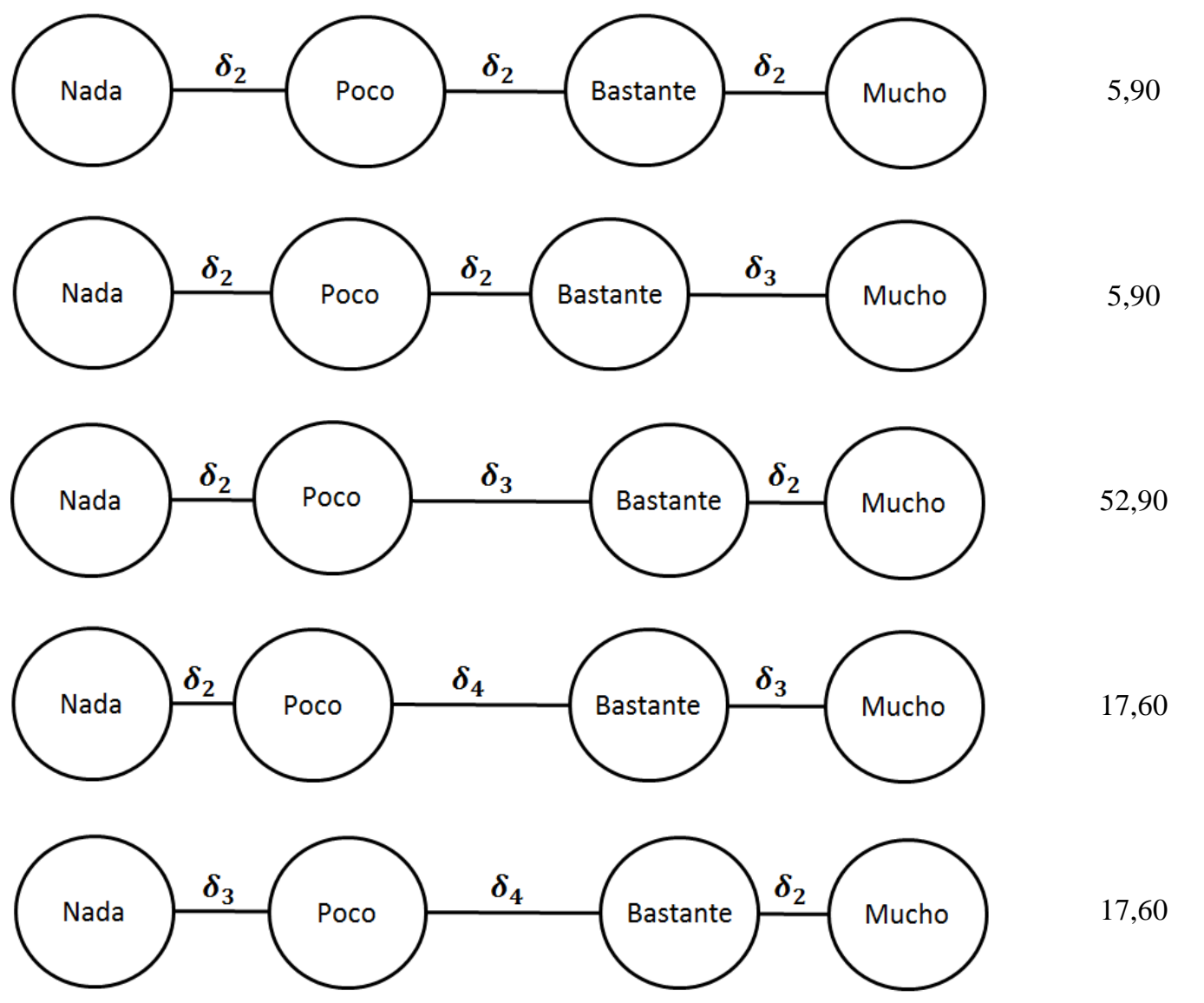

Fuente: Elaboración propia a partir de las respuestas de la encuesta realizada. $N=20$.

Tras la aplicación de este procedimiento se obtiene la medida de proximidad ordinal representada en la Figura 3, donde puede apreciarse que existe la misma proximidad entre las categorías de respuesta "Nada” - "Poco” y entre "Bastante” - "Mucho” $\left(\delta_{2}\right)$, que es mayor que la proximidad existente entre "Poco" - "Bastante" $\left(\delta_{3}\right)$.

Figura 3. Medida de proximidad ordinal considerada para la aplicación del procedimiento ordinal.

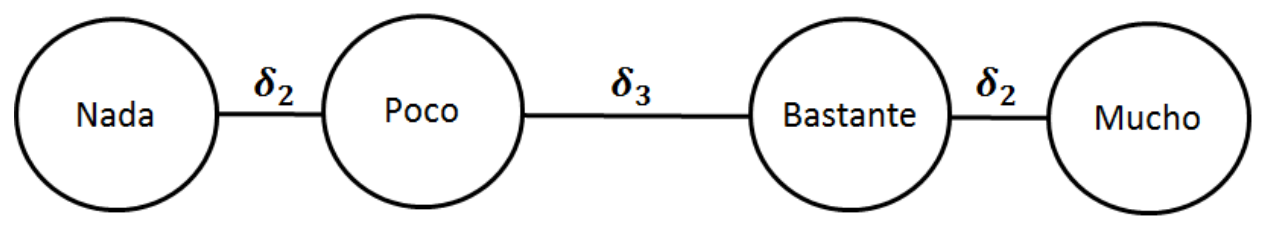

Fuente: Elaboración propia.

Finalmente, los resultados obtenidos después de ordenar las nueve alternativas mediante la agrupación de las categorías de respuesta “Mucho” y “Bastante” y la aplicación del procedimiento ordinal de la sección 2 se muestran en la Tabla 7. Los diferentes órdenes obtenidos se presentan desagregados por sexo, tal y como suele realizar el CIS en sus anuarios cuando publica los resultados de sus diversos estudios. 
Tabla 7. Órdenes obtenidos tras la agrupación de "Mucho” y "Bastante” y la aplicación del procedimiento ordinal.

\begin{tabular}{|c|c|c|c|c|c|}
\hline \multicolumn{6}{|c|}{ Agrupación de "Mucho" y "Bastante" } \\
\hline & Total & & Hombres & & Mujeres \\
\hline 1 & Sus familiares & 1 & Los bomberos & 1 & Sus familiares \\
\hline 2 & Los bomberos & 2 & Sus familiares & 2 & Los bomberos \\
\hline 3 & Sus amigos & 3 & Sus amigos & 3 & Sus amigos \\
\hline 4 & Protección Civil & 4 & Protección Civil & 4 & Protección Civil \\
\hline 5 & El ejército & 5 & El ejército & 5 & El ejército \\
\hline 6 & La policía & 6 & La Guardia Civil & 6 & La policía \\
\hline 7 & La Guardia Civil & 7 & La policía & 7 & La Guardia Civil \\
\hline 8 & Sus vecinos & 8 & Sus vecinos & 8 & Sus vecinos \\
\hline 9 & Las ONGs & 9 & Las ONGs & 9 & Las ONGs \\
\hline \multicolumn{6}{|c|}{ Procedimiento ordinal } \\
\hline & Total & & Hombres & & Mujeres \\
\hline & Sus familiares & & Sus familiares & 1 & Sus familiares \\
\hline 2 & Los bomberos & 2 & Los bomberos & 2 & Los bomberos \\
\hline 3 & Sus amigos & 3 & Sus amigos & 3 & Sus amigos \\
\hline 4 & El ejército & 4 & El ejército & 4 & Protección Civil \\
\hline 5 & Protección Civil & 5 & Protección Civil & 5 & El ejército \\
\hline & La Guardia Civil & 6 & La Guardia Civil & 6 & La Guardia Civil \\
\hline 7 & La policía & 7 & La policía & 7 & La policía \\
\hline & Sus vecinos & 8 & Sus vecinos & 8 & Sus vecinos \\
\hline 9 & Las ONGs & 9 & Las ONGs & 9 & Las ONGs \\
\hline
\end{tabular}

Fuente: Elaboración propia.

Observando los resultados de la Tabla 7, en primer lugar cabe señalar que no existen diferencias en las posiciones ocupadas por las alternativas vecinos y ONGs, que ocupan en todos los órdenes de la tabla la séptima y octava posición, respectivamente. En cambio, sí que existen diferencias en la forma de ordenar el resto de alternativas.

Si se considera la población general, sin hacer distinciones de género, las diferencias entre los órdenes obtenidos mediante la agrupación de las categorías y la aplicación del procedimiento ordinal se encuentran en las alternativas Protección Civil, ejército, Policía y Guardia Civil.

Protección Civil y el ejército, que ocupan la cuarta y la quinta posición en el orden resultante de la agrupación de "Mucho" y "Bastante”, invierten sus posiciones en el caso del orden obtenido tras aplicar el procedimiento ordinal, sucediendo lo mismo en el caso de la Policía y la Guardia Civil.

En cuanto a los hombres, existen diferencias significativas en las primeras posiciones de los órdenes. Los bomberos y los familiares ocupan la primera y la segunda posición, respectivamente, en el orden obtenido tras la agrupación de las categorías. Sin embargo, si se 
considera el procedimiento ordinal, los familiares se sitúan en la primera posición seguidos por la alternativa bomberos. Las alternativas Protección Civil y el ejército también sufren modificaciones, invirtiéndose las posiciones que ocupan en los órdenes resultantes.

En el caso de las mujeres, las diferencias entre los dos órdenes son poco relevantes, alterándose únicamente las posiciones de las alternativas Policía y Guardia Civil.

\section{Conclusiones.}

El uso de escalas cualitativas formadas por términos lingüísticos permite a los seres humanos expresar sus valoraciones y juicios subjetivos de una forma sencilla ante situaciones de vaguedad e imprecisión. Desde un punto de vista práctico es muy frecuente que las escalas cualitativas se representen a través de valores numéricos exactos, debido a las facilidades que proporciona la Estadística a la hora de manejar datos numéricos.

No obstante, este enfoque cuantitativo de las escalas cualitativas desvirtúa la propia naturaleza de las mismas, ya que contradice algunos principios de la Teoría de la Medición (Stevens, 1951; Roberts, 1979), llegando incluso a generar problemas de interpretación de los resultados procedentes de las escalas cualitativas no uniformes.

Por otra parte, la práctica utilizada por diversos organismos consistente en analizar y presentar los resultados de sus investigaciones mediante la agrupación de varias categorías tampoco está considerando la no uniformidad de las escalas cualitativas, suponiendo además una pérdida importante de información cualitativa al descartar parte de las categorías de respuesta de las escalas.

En este trabajo se ha realizado un análisis de la metodología de agrupación de varias categorías de respuesta que utilizada el CIS, considerando como método alternativo a dicha práctica el procedimiento ordinal introducido por García-Lapresta y Pérez-Román (2018).

Para la aplicación del procedimiento ordinal fue necesario establecer una medida de proximidad ordinal. Con el objeto de establecer dicha medida y conocer cómo aprecian los individuos las proximidades entre los términos de la escala \{ "Nada", "Poco", "Bastante", "Mucho" \}, se realizó una encuesta a un grupo de estudiantes. Los datos de la misma evidenciaron la no uniformidad de la escala, ya que un $94,10 \%$ de los estudiantes percibió la escala del CIS como no uniforme.

Por otra parte, respecto a las modificaciones y extensiones futuras de este procedimiento, puede señalarse la propuesta introducida recientemente por García-Lapresta y González del Pozo (2019) en contextos de duda. Dicho procedimiento es una extensión del procedimiento ordinal que puede aplicarse en escenarios en los que existen múltiples criterios y a los individuos se les permite seleccionar dos términos consecutivos de la escala.

\section{Agradecimientos}

Los autores agradecen a los evaluadores anónimos sus comentarios y sugerencias que nos han permitido mejorar el trabajo, así como al Ministerio de Economía y Competitividad (MINECO) y al Fondo Europeo de Desarrollo Regional (FEDER) (referencia ECO2016-77900-P) por la financiación recibida. 


\section{Referencias}

Balinski, M., \& Laraki, R. (2007). A theory of measuring, electing and ranking. Proceedings of the National Academy of Sciences of the United States of America, 104, 8720-8725.

Balinski, M., \& Laraki, R. (2011). Majority Judgment: Measuring Ranking and Electing. Massachusetts: MIT Press.

Beyth-Marom, R. (1982). How probable is probable? A numerical taxonomy translation of verbal probability expressions. Journal of Forecasting, 1, 257-269.

Díaz de Rada, V. (2004). Problemas de representatividad en las encuestas que utilizan muestreos probabilísticos. Papers, 74, 45-66.

Díaz de Rada, V. (2015). Calidad de los datos de preguntas de batería en encuestas presenciales: una comparación de un estudio con cuestionario en papel y en formato electrónico. Revista Española de Investigaciones Sociológicas, 152, 167-177.

Durán, A., Ocaña, A.C., Cañadas, I., \& Pérez, F.J. (2000). Construcción de cuestionarios para encuestas: el problema de la familiaridad de las opciones de respuesta. Metodología de Encuestas, 2(1), 27-60.

Epstein, L.G. (1999). A definition of uncertainty aversion. The Review of Economic Studies, 66(3), 579-608.

Fleiss, J.L., Levin, B., \& Paik, M.C. (2003). Statistical Methods for Rates and Proportions. New Jersey: Wiley and Sons.

Franceschini, F., Galetto, M., \& Varetto, M. (2004). Qualitative ordinal scales: the concept of ordinal range. Quality Engineering, 16, 515-524.

García-Lapresta, J.L., \& González del Pozo, R. (2019). An ordinal multi-criteria decision-making procedure under imprecise linguistic assessments. European Journal of Operational Research, 279, 159-167.

García-Lapresta, J.L., González del Pozo, R., \& Pérez-Román, D. (2018). Metrizable ordinal proximity measures and their aggregation. Information Sciences, 448-449, 149-163.

García-Lapresta, J.L., \& Pérez-Román, D. (2015). Ordinal proximity measures in the context of unbalanced qualitative scales and some applications to consensus and clustering. Applied Soft Computing, 35, 864-872.

García-Lapresta, J.L., \& Pérez-Román, D. (2018). Aggregating opinions in non-uniform ordered qualitative scales. Applied Soft Computing, 67, 652-657.

Grossi, D., \& Pigozzi, G. (2014). Judgment Aggregation: A Primer. Synthesis Lectures on Artificial Intelligence and Machine Learning. Morgan and Claypool.

Kennedy, R., Riquier, C., \& Sharp, B. (1996). Practical applications of correspondence analysis to categorical data in market research. Journal of Targeting, Measurement and Analysis for Marketing, 5(1), 56-70. 
Likert, R. (1932). A technique for the measurement of attitudes. Archives of Psychology, 22(140), 1-55.

Lozano, L.M., García-Cueto, E., \& Muñiz, J. (2008). Effect of the number of response categories on the reliability and validity of rating scales. Methodology, 4, 73-79.

Mangione, T.W. (1995). Mail Surveys: Improving the Quality. Applied Social Research Methods. Thousand Oaks: Sega Publications.

Matas, A. (2018). Diseño del formato de escalas tipo Likert: un estado de la cuestión. Revista Electrónica de Investigación Educativa, 20(1), 38-47.

Merbitz, C., Morris, J., \& Grip, J.C. (1989). Ordinal scales and foundations of misinference. Archives of Physical Medicine and Rehabilitation, 70, 308-312.

Nadler, J., Weston, R., \& Voyles, E. (2015). Stuck in the middle: the use and interpretation of mid-points in items on questionnaires. The Journal of General Psychology, 142(2), 7189.

Pardo, A. (2002). Análisis de Datos Categóricos. Madrid: UNED.

Roberts, F.S. (1979). Measurement Theory. New York: Cambridge University Press.

Silva, L.C. (1997). Cultura Estadística e Investigación Científica en el Campo de la Salud: una Mirada Crítica. Madrid: Ediciones Díaz de Santos.

Stevens, S.S. 1951. Mathematics, Measurement and Psychophysics. Oxford, England: Wiley.

Teigen, K.H., \& Wibecke, B. (1999). The directionality of verbal probability expressions: effects on decisions, predictions, and probabilistic reasoning. Organizational Behavior and Human Decision Processes, 80(2), 155-190.

Zimmer, A.C. (1983). Verbal vs. numerical processing by subjective probabilities. En: Scholz, R.W. (ed.). Decision Making under Uncertainty, pp. 159-182. North Holland, Amsterdam. 


\section{ANEXO. Ejemplo de aplicación del procedimiento ordinal.}

1. Recoger las valoraciones cualitativas $v_{i}^{a}$ dadas por cinco individuos $A=\{1,2,3,4,5\}$ sobre cada una de las alternativas $X=\left\{x_{1}, x_{2}, x_{3}\right\}$. En este ejemplo se ha utilizado la escala cualitativa ordenada $\mathcal{L}=\left\{l_{1}, l_{2}, l_{3}, l_{4}\right\}$, donde $l_{1} \prec l_{2} \prec l_{3} \prec l_{4}$.

Tabla 8. Valoraciones cualitativas dadas por cinco individuos a las alternativas $x_{1}, x_{2}$ y $x_{3}$.

\begin{tabular}{ccc}
\hline $\boldsymbol{x}_{\mathbf{1}}$ & $\boldsymbol{x}_{\mathbf{2}}$ & $\boldsymbol{x}_{\mathbf{3}}$ \\
\hline$l_{3}$ & $l_{2}$ & $l_{1}$ \\
$l_{1}$ & $l_{3}$ & $l_{2}$ \\
$l_{3}$ & $l_{4}$ & $l_{2}$ \\
$l_{4}$ & $l_{3}$ & $l_{3}$ \\
$l_{3}$ & $l_{4}$ & $l_{4}$ \\
\hline
\end{tabular}

Fuente: Elaboración propia.

2. Establecer sobre la escala cualitativa una medida de proximidad ordinal adecuada $\pi: \mathcal{L}^{2} \rightarrow \Delta$. En este ejemplo hemos considerado la medida de proximidad ordinal representada en la Figura 4.

Figura 4. Medida de proximidad ordinal usada en el ejemplo.

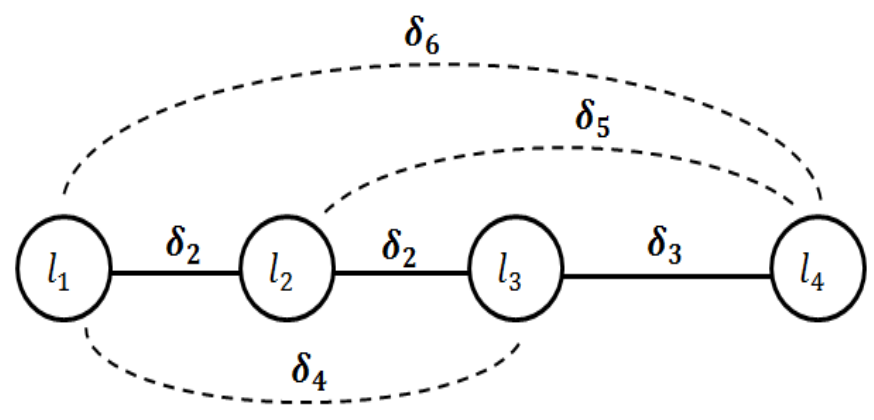

Fuente: Elaboración propia.

3. Para cada alternativa $x_{i} \in X$ se calculan los grados de proximidad entre las valoraciones cualitativas dadas por los individuos y la máxima valoración posible de la escala, $l_{4}$. 
Tabla 9. Grados de proximidad obtenidos tras comparar cada una de las valoraciones cualitativas con la máxima valoración de la escala.

\begin{tabular}{ccc}
\hline $\boldsymbol{x}_{\mathbf{1}}$ & $\boldsymbol{x}_{\mathbf{2}}$ & $\boldsymbol{x}_{\mathbf{3}}$ \\
\hline$\delta_{3}$ & $\delta_{5}$ & $\delta_{6}$ \\
$\delta_{6}$ & $\delta_{3}$ & $\delta_{5}$ \\
$\delta_{3}$ & $\delta_{1}$ & $\delta_{5}$ \\
$\delta_{1}$ & $\delta_{3}$ & $\delta_{3}$ \\
$\delta_{3}$ & $\delta_{1}$ & $\delta_{1}$ \\
\hline
\end{tabular}

Fuente: Elaboración propia.

4. Ordenar para cada alternativa $x_{i} \in X$ los grados de proximidad de forma decreciente (de mayor a menor proximidad) y seleccionar para cada alternativa su par de medianas, $M_{i} \in \Delta_{2}$, donde $\Delta_{2}$ es el conjunto de medianas factibles:

$$
\Delta_{2}=\left\{\left(\delta_{r}, \delta_{s}\right) \in \Delta^{2} \mid r \leq s\right\}
$$

Tabla 10. Medianas de los grados de proximidad.

\begin{tabular}{ccc}
\hline $\boldsymbol{x}_{1}$ & $\boldsymbol{x}_{2}$ & $\boldsymbol{x}_{3}$ \\
\hline$\delta_{1}$ & $\delta_{1}$ & $\delta_{1}$ \\
$\delta_{3}$ & $\delta_{1}$ & $\delta_{3}$ \\
$\delta_{3}$ & $\delta_{3}$ & $\delta_{5}$ \\
$\delta_{3}$ & $\delta_{3}$ & $\delta_{5}$ \\
$\delta_{6}$ & $\delta_{5}$ & $\delta_{6}$ \\
\hline
\end{tabular}

Fuente: Elaboración propia.

Como en este caso, el número de grados de proximidad es impar, se duplica la mediana. Por tanto, $M_{1}=M_{2}=\left(\delta_{3}, \delta_{3}\right)$ y $M_{3}=\left(\delta_{5}, \delta_{5}\right)$.

5. Establecer un orden lineal apropiado sobre $\Delta_{2}$ para ordenar los pares de medianas de los grados de proximidad.

6. Los pares de medianas se ordenan siguiendo el orden lineal introducido por García-Lapresta y Pérez-Román (2018):

$$
\begin{gathered}
\left(\delta_{r}, \delta_{s}\right) \succcurlyeq_{2}\left(\delta_{t}, \delta_{u}\right) \Leftrightarrow\left\{\begin{array}{l}
r+s<t+u \\
0 \\
r+s=t+u
\end{array} \quad \mathrm{y} \quad s-r \leq u-t\right. \\
\left(\delta_{2}, \delta_{2}\right)>_{2}\left(\delta_{1}, \delta_{3}\right)>_{2}\left(\delta_{2}, \delta_{3}\right)>_{2}\left(\delta_{1}, \delta_{4}\right)>_{2}\left(\delta_{3}, \delta_{3}\right)>_{2}\left(\delta_{2}, \delta_{4}\right)>_{2} \cdots
\end{gathered}
$$


7. Ordenar las alternativas teniendo en cuenta el orden débil sobre $X$ definido como $x_{i} \geqslant x_{j} \Leftrightarrow M_{i} \geqslant_{2} M_{j}$, y aplicar el procedimiento de desempate si fuera necesario.

Teniendo en cuenta el orden establecido en el paso 6, $M_{1}=M_{2}>_{2} M_{3} ; x_{1}>x_{3}$ y $x_{2}>x_{3}$.

Puesto que las alternativas $x_{1}$ y $x_{3}$ tienen la misma mediana, se aplica el procedimiento de desempate. Para ello se eliminan las medianas de las alternativas $x_{1}$ y $x_{3}$ y se vuelven a calcular sus medianas entre los grados de proximidad restantes.

Tabla 11. Procedimiento de desempate.

\begin{tabular}{cc}
\hline $\boldsymbol{x}_{\mathbf{1}}$ & $\boldsymbol{x}_{\mathbf{2}}$ \\
\hline$\delta_{1}$ & $\delta_{1}$ \\
$\delta_{3}$ & $\delta_{1}$ \\
$\delta_{3}$ & $\delta_{3}$ \\
$\delta_{3}$ & $\delta_{3}$ \\
$\delta_{6}$ & $\delta_{5}$
\end{tabular}

\begin{tabular}{cc}
\hline $\boldsymbol{x}_{\mathbf{1}}$ & $\boldsymbol{x}_{\mathbf{2}}$ \\
\hline$\delta_{1}$ & $\delta_{1}$ \\
$\delta_{3}$ & $\delta_{1}$ \\
$\delta_{3}$ & $\delta_{3}$ \\
$\delta_{6}$ & $\delta_{5}$ \\
\hline
\end{tabular}

Fuente: Elaboración propia.

$$
M_{1}^{(1)}=\left(\delta_{3}, \delta_{3}\right) \text { y } M_{2}^{(1)}=\left(\delta_{1}, \delta_{3}\right)
$$

Teniendo en cuenta el orden introducido en el paso $6, M_{2}^{(1)}>_{2} M_{1}^{(1)}$ y, por tanto, $x_{2}>x_{1}$.

Finalmente, y tras la aplicación del proceso de desempate, se obtiene el siguiente resultado: $x_{2}>$ $x_{1}>x_{3}$. 